\title{
Tratamiento de sustitución con Metadona para la adicción a la Heroína: evaluación de un programa en Colombia
}

\section{Methadone substitution treatment for heroin addiction: a program assessment in Colombia}

\author{
| Mario A. ZapatA*; LUIS F. GIRALDo**
}

${ }^{*}$ NIDA Fellow - Grupo de Investigación en Salud Mental, Universidad CES.
** CARISMA, Centro de Atención y Rehabilitación en Salud Mental de
Antioquia - Colombia.

Enviar correspondencia a:

Mario A. Zapata

Universidad CES-Facultad de Medicina.

Grupo de Investigación en Salud Mental.

Calle 10A \#22-04 Medellín, Colombia (SA).

mzapatav@une.net.co

\section{Resumen}

Evaluación de un programa de tratamiento de sustitución y mantenimiento con metadona en Colombia mediante aplicación del instrumento OTI (Opiate Treatment Index). Estudio descriptivo que utiliza muestra de 50 pacientes atendidos en 2007-2008 para la adicción a heroína. Con el instrumento OTI se evalúan áreas de consumo de drogas, conductas de riesgo para el contagio de $\mathrm{VIH}$, funcionamiento social, actividad delictiva, estado de salud y ajuste psicológico. El 74\% de la muestra son hombres y 26\% mujeres, con edad media 22 años (64\% pertenece al grupo 20-24 años). La edad de inicio en el consumo de heroína es 18 años (rango entre 13 y 28). En el 38\% (19 de 50) se encontró comorbilidad con otros trastornos mentales, siendo el $47 \%$ trastornos de personalidad. La dosis actual de metadona es $40 \mathrm{mg} / \mathrm{dia}$, rango entre 15 y $80 \mathrm{mg} / \mathrm{dia}$.

Las escalas del instrumento OTI muestran conducta de riesgo para VIH en nivel "Bajo-Medio"; funcionamiento social, escala delictiva y estado de salud riesgo "Bajo"; la escala de ajuste psicológico muestra riesgo "BajoMedio" en el grupo 0-12 meses y "Medio" en los grupos 12-24 y >24 meses; para todas las diferencias entre grupos y riesgos no se encontró significancia estadistica.

El consumo de heroína en esta población inicia a edad temprana. En general el riesgo evaluado en las diferentes áreas del OTI es bajo, posiblemente por la etapa inicial de consumo problemático de heroina en Colombia. Existe alta comorbilidad con trastornos de personalidad.

Palabras clave: adicción heroína, tratamiento de sustitución, metadona, OTI. recibido: Marzo 2011 aceptado: Diciembre 2012

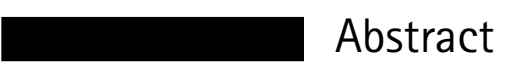

Assessment of a methadone substitution and maintenance treatment program in Colombia using the OTI (Opiate Treatment Index) instrument. Descriptive study with a 50-patient sample attended in the period 20072008 for their heroin addiction. The OTI instrument was implemented to assess drug use, HIV risk-taking behavior, social functioning, criminality, health status and psychological functioning. Men accounted for $74 \%$ of the sample, women for $26 \%$. Mean age was 22, with 64\% in the $20-24$ age group. Mean reported age at onset of heroin use was 18 , with a range of 13-28. In 38\% of the sample (19 patients) comorbidity with other mental disorders was found, $47 \%$ of these being personality disorders. Median reported current methadone dose was $40 \mathrm{mg} / \mathrm{day}$, with a range of $15-80 \mathrm{mg} / \mathrm{day}$.

According to the OTI subscales, HIV risk-taking behavior was low-moderate, while social functioning, criminality and health status were low-risk. In the case of psychological adjustment, a low-moderate risk score was obtained for the 0-12 month treatment group, and a moderate risk score for the $12-24$ and $\geq 24$ month groups.

Starting age for heroin use in this population is early. In general, the risk assessed in the OTI areas is low, possibly due to problematic heroin use being in its early stages in Colombia, but comorbidity with personality disorders is high.

Key words: heroin addiction, substitution therapy, methadone, Opiate Treatment Index. 
$\mathrm{D}$ esde el año 2002 se dio la señal de alarma en Colombia (Sur América) por la aparición en cantidades inesperadas de nuevos pacientes dependientes a la heroína en los centros de tratamiento, en contraposición a la epidemiología del consumo de sustancias ilegales en esta región (Castaño y Calderón, 2010; Zapata, 2004). Esta situación fue mencionada también en el informe anual de las Naciones Unidas donde se afirma, "Sur América tiene el más bajo nivel de abuso en el mundo pero existen indicadores que muestran incremento en el uso de heroína" (UNODC, 2004).

Para atender este problema, la institución estatal de tratamiento en adicciones "Carisma" (Centro de Atención y Rehabilitación en Salud Mental de Antioquia), elaboró un protocolo de tratamiento para la dependencia de opiáceos a partir del proyecto "Treatnet" (2006) de la Oficina de las Naciones Unidas contra las Drogas y el Delito y teniendo en cuenta también los modelos de tratamiento de Australia y Canadá (Correctional Service of Canada, 2003; Henry-Edwards, Gowing, White, Ali, Bell, Brough et al., 2003). Iniciativas como esta enfrentan el reto de generar cambios en paradigmas de tratamiento, con el propósito de incorporar la reducción del daño y sus beneficios en los modelos de atención primaria (Erickson, 1995; Fiellin, 0'Connor, Chawarski, Pakes, Pantalon y Schottenfeld, 2001; Krantz y Mehler, 2004; Langendam, van Brussel, Coutinho y van Ameijden, 2001; Trujols et al., 2010). Adoptar nuevas formas de tratamiento en paises de Suramérica en donde el consumo de heroína no ha sido un problema mayor en el pasado (UNODC, 2010), enfrenta dificultades con la cultura de estos paises, con las prioridades en salud pública existentes y con el marco normativo vigente. Todo ello puede dificultar el desarrollo de protocolos en donde se priorice la reducción del daño. Se requieren consensos entre profesionales e instituciones que faciliten su aceptación, como el logrado en los Estados Unidos (NIH Consensus Conference, 1998).

Otro paso importante es la implementación de metodologías de evaluación de tratamientos que demuestren su efectividad. El instrumento de evaluación OTI - Opiate Treatment Index desarrollado por Darke, Hall, Wodak, Heather y Ward (1992) y validado en España por González-Saiz, SalvadorCarulla, Martínez-Delgado, López-Cárdenas, Ruz-Franzi y Guerra-Díaz (1997), ha sido utilizado en otros estudios en el mundo (Adelekan, Green, Dasgupta, Tallack, Stimson y Wells, 1996; Deering y Sellman, 1996) y en él se tienen en cuenta características que van más allá de la cesación o no del consumo, siendo considerado un instrumento multidimensional al evaluar seis dominios.

En el presente artículo se presentan los resultados de la evaluación del tratamiento de 50 pacientes con dependencia de heroína en el inicio de un programa de mantenimiento con metadona (PMM) en Colombia. Los objetivos de esta investigación pretenden caracterizar la población del nuevo programa y evaluar por primera vez los resultados del tratamiento mediante la aplicación del instrumento de medición OTI, lo cual puede ser útil para la implementación de programas similares en el país y en la región Suramericana.

\section{Método}

\section{Población y muestra}

La muestra está compuesta por 50 pacientes en tratamiento de sustitución con metadona para su problema de adicción a heroína, según criterios del DSM IV-TR, que corresponden al 70\% del total de 72 usuarios que ingresaron durante los años 2007-2008 para tratamiento en nuestro Centro, siendo evaluados mediante el OTI en su versión española.

\section{Instrumentos}

El instrumento "Indicador del Tratamiento de la Adicción a Opiáceos" (González-Saiz, et al. 1997), versión española de la entrevista clínica "Opiate Treatment Index - OTI" desarrollado y validado por Darke, et al. (1992), fue utilizado con la autorización de sus autores previa adaptación al lenguaje de la región y a la situación particular del consumo de heroína en Colombia. El OTI es una entrevista estructurada que mide específicamente 6 áreas: consumo de drogas, conducta de riesgo de contagio de V.I.H., funcionamiento social, actividad delictiva, estado de salud y ajuste psicológico, esta última incorporada al OTI de la escala General Health Questionnaire de Goldberg y Williams (1988).

Para todas las subescalas del OTI una mayor puntuación se interpreta como mayor disfunción en el área evaluada; para disminuir el sesgo de memoria y medir de manera más próxima la realidad del paciente entrevistado, todas las subescalas con excepción del funcionamiento social se refieren al mes anterior de la entrevista. La subescala funcionamiento social hace referencia a los últimos 6 meses y para este estudio fue adaptada la variable original del instrumento "numero de trabajos", en consideración a la necesidad de evaluar la calidad de ser estudiante, situación que es común en esta muestra. Se diseñó entonces una variable adicional para no afectar la medición total del instrumento validado, incorporando una nueva subescala para estudiantes y extraída del DUSI - Drug Use Screening Inventory de su área problemas escolares (Tarter, Kirisci y Mezzich, 1996); se equiparó con los 5 niveles de riesgo de la variable original: 0 , sin problemas; 1 , problemas leves; 2 y 3 , problemas moderados; 4 o más, problemas graves.

\section{Procedimiento}

La recolección de la información es realizada por médico entrenado en el manejo del instrumento, mediante entrevista programada con los usuarios atendidos en los años 2007 y 2008, previo proceso de consentimiento informado. La información es introducida en y analizada en el programa estadistico SPSS. 
En una primera etapa se obtienen medidas descriptivas de la población estudiada, comparando los resultados en 3 grupos temporales de tratamiento, 0-12 meses, 12 a 24 y mayor a 24 meses. Previa verificación de la normalidad de la muestra se utilizó la prueba no paramétrica de KruskalWallis, con un nivel de confianza del 95\%.

\section{Resultados}

\section{Características Sociodemográficas}

La distribución por sexo (tabla 1) muestra que el 74\% son hombres; la edad media de los pacientes es de 22 años y el $64 \%$ se encuentra en el grupo de 20 a 24 . El nivel educativo de los pacientes tiene una media de 11 años y el $72 \%$ tiene estudios de secundaria.

La población estudiada es descrita en nivel de pobreza intermedio-bajo (nivel 2) para el 40\%, que sumados a los niveles de pobreza 0 y 1 , según la metodología de clasificación utilizada en Colombia para sus programas sociales, suman un porcentaje del $60 \%$ del total. Con respecto al seguro de salud, el $72 \%$ pertenece al esquema de subsidios estatales, probable consecuencia del carácter público de la institución donde se realizó el estudio.

Tabla 1. Características de los pacientes $(\mathrm{N}=50)$ en el programa de mantenimiento con Metadona

\begin{tabular}{llcc}
\hline & Variable & N & $\%$ \\
\hline \multirow{2}{*}{ Sexo } & Hombre & 37 & 74 \\
& Mujer & 13 & 26 \\
\hline Edad Agrupada & $15-19$ años & 6 & 12 \\
$x=23,2$ & $20-24$ años & 32 & 64 \\
$M e=22$ & $25-29$ años & 7 & 14 \\
Mo=22 & $30-34$ años & 5 & 10 \\
\hline Nivel educativo & Primaria & 3 & 6 \\
$x=10,2$ & Secundaria & 36 & 72 \\
Me=11 & Técnica y Superior & 11 & 22 \\
\hline No=11 & Nivel 0 & 3 & 6 \\
& Nivel 1 & 7 & 14 \\
& Nivel 2 & 20 & 40 \\
& Nivel 3 & 7 & 14 \\
& Otro con capacidad de pago & 13 & 26 \\
\hline Aseguramiento en Salud & Vinculado & 0 & 0 \\
& Subsidiado & 36 & 72 \\
& Contributivo & 14 & 28 \\
\hline
\end{tabular}

\section{Antecedentes del tratamiento}

- Droga de inicio en el consumo de sustancias psicoactivas

La marihuana es la droga ilegal de inicio más frecuente con 35 de los 50 pacientes (70\%), le sigue la cocaína con 10 (20\%), en tercer lugar inhalables $3(6 \%)$ y sólo 1 paciente se inició con heroína (2\%).
- Edad de inicio consumo de drogas

La edad media y mediana para el consumo de drogas ilegales fue de 14 años, con un rango de inicio entre los 9 y los 21 años; con respecto al inicio del consumo de heroína la media y la mediana es 18 años con un rango entre 13 y 28.

\section{- Número de tratamiento previos}

Se indagó el número de tratamientos previos para la dependencia a la heroína, encontrándose una mediana de 2 y un rango entre 2 y 6 tratamientos previos.

\section{- Dosis de metadona en tratamientos previos y actual}

Con respecto a la dosis de metadona utilizada en el tratamiento actual y en los tratamientos previos, se encontró una mediana en el tratamiento más antiguo (tratamiento 2) de 25 $\mathrm{mg} /$ día con un rango entre 20 y $30 \mathrm{mg} /$ día. Para el tratamiento anterior (tratamiento 1), se reporta una mediana de $40 \mathrm{mg} /$ día, rango entre 15 y $80 \mathrm{mg} /$ dia y en el tratamiento actual se encuentra una mediana de $40 \mathrm{mg} / \mathrm{día}$, rango entre $10 \mathrm{y}$ $100 \mathrm{mg} / \mathrm{dia}$.

Se resalta el incremento de la mediana de $15 \mathrm{mg} /$ día entre el tratamiento anterior 2 y el tratamiento anterior 1 y ningún incremento entre el tratamiento anterior 1 y el tratamiento actual; igualmente se observa un importante incremento en el rango máximo entre tratamientos previos y actual, siendo en el primer tratamiento de $30 \mathrm{mg} /$ día y en el actual de $100 \mathrm{mg} /$ día.

\section{Diagnósticos al alta y comorbilidad en salud mental}

El diagnóstico de dependencia a la heroína como primer y único diagnóstico al alta en el programa evaluado, es reportado en 27 de los 50 pacientes (54\%) y en el 46\% restante se diagnosticó comorbilidad con la siguiente distribución: dependencia a la cocaína 3(6\%), epilepsia $1(2 \%)$ y trastornos mentales 19(38\%). Dentro de estos últimos se destacan los trastornos de personalidad (9 casos), lo cual corresponde a $47 \%$ del total de los trastornos mentales y al $18 \%$ del total de la población evaluada (gráfico 1).

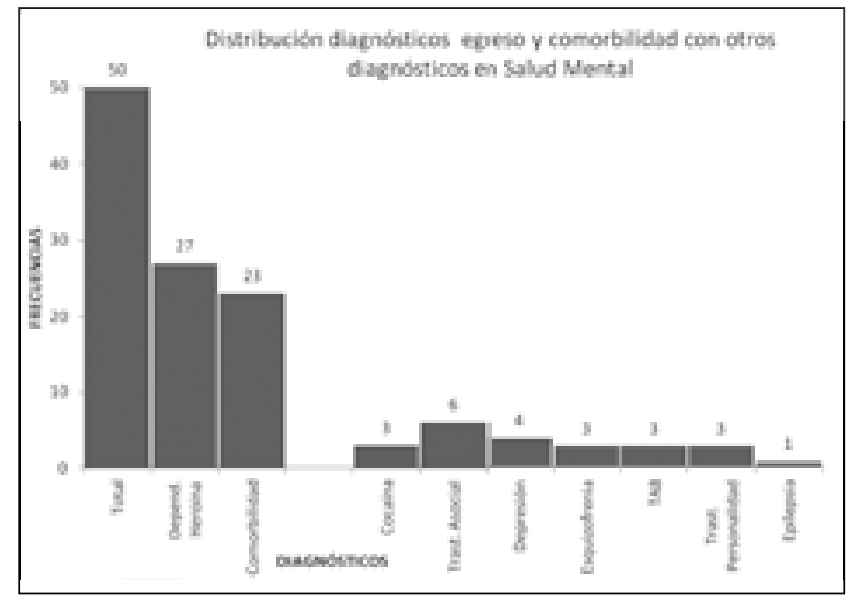

Evaluación de las escalas de riesgo del instrumento OTI

Gráfico 1. Diagnóstico principal y otros diagnósticos en salud mental 


\section{- Consumo de drogas}

Para el consumo de drogas incluyendo alcohol y tabaco, los resultados presentados en la tabla 2 no muestran diferencias estadísticamente significativas para los niveles de riesgo en los 3 grupos temporales de tratamiento evaluados $(p=0,628)$. En busca de evaluar el efecto que el consumo de sustancias legales tiene para la puntuación de riesgo en esta área del OTI, se excluyen el alcohol y el tabaco para observar el resultado de la medición del riesgo sin estas sustancias; adicionalmente se excluye de manera individual el alcohol para un análisis y tabaco para el otro. Acorde a los resultados (tabla 2), puede observarse que el riesgo de consumo de drogas es menor cuando se excluye del análisis el tabaco y el alcohol, se presentan valores similares de riesgo alto cuando se excluye del análisis el alcohol y se observa en los tres grupos valores de riesgo bajo cuando se excluye solamente el tabaco. Lo anterior muestra que el consumo de tabaco soporta en gran medida los valores altos de este indicador de riesgo; en otras palabras, el tabaco, a la luz de este resultado, es la sustancia psicoactiva que predomina en el consumo para esta cohorte de pacientes.

De manera general se observa que el indicador de riesgo de consumo de drogas es menor en los pacientes que llevan más tiempo en tratamiento $(>24$ meses $=4,97)$ comparado con el resultado de 6,10 de 0-12 meses y 5,52 de 12-24 meses; cuando se excluye la influencia que produce el consumo de tabaco para este indicador, se encuentra que el valor es superior en el grupo de 12 a 24 meses con un resultado de 2,2 (tabla 2).

\section{Conductas de riesgo de contagio de/ VIH}

Los resultados encontrados para el riesgo de consumo de drogas vía parenteral con la utilización y reutilización de agujas y jeringas, muestran un aumento en la calificación de riesgo entre los grupos, pero las diferencias no fueron significativas con valor de $p=0,685$ (tabla 2). Con respecto al riesgo de la conducta y hábitos sexuales, se encuentra una mayor calificación de riesgo en el grupo mayor a 24 meses de tratamiento pero sin significancia estadistica (valor de $p=0,915)$.

De manera general se observa que la calificación de la escala de riesgo para las conductas favorecedoras de infección por VIH son crecientes a mayor tiempo de tratamiento, pero este aumento en el riesgo de contagio de VIH depende en una gran proporción del riesgo asociado a la conducta sexual (total conducta sexual $=4,02$ y total riesgo asociado al consumo de drogas=1,10). La categoría clínica de gravedad según la puntuación de la escala OTI para el riesgo de $\mathrm{VIH}$, fue baja-media para los tres grupos de tratamiento; las diferencias entre grupos no fueron estadísticamente significativas $(p=0,745)$.

\section{Funcionamiento social}

Esta escala presenta como resultado una calificación de riesgo total de 12,22 puntos y se observa una disminución en la calificación de riesgo cercana a 2 puntos, comparados el grupo de 0-12 meses de tratamiento $(13,43)$ vs. los grupos 12-24 y mayor a 24 meses pero las diferencias no fueron estadísticamente significativas $(p=0,403)$. La categoría clínica de gravedad según la puntuación de la escala OTI para esta área de riesgo fue baja para los tres grupos de tratamiento evaluados.

\section{Actividad delictiva}

En esta subescala se encuentran valores muy bajos cercanos a cero para el total de pacientes y en los tres grupos evaluados; las diferencias encontradas entre grupos no muestran significancia estadistica $(p=0,60)$.

La categoría clínica de gravedad para esta área de riesgo fue baja para el total y los tres grupos de tratamiento evaluados.

\section{Estado de Salud}

El valor total de riesgo para el estado de salud es bajo $(4,92)$; En esta subescala se encuentra que a mayor tiempo en el tratamiento menor calificación de riesgo, con diferencias que no son estadísticamente significativas $(p=0,930)$.

\section{Ajuste psicológico, GHO-28}

Los resultados de ajuste psicológico muestran mayor puntaje en la calificación del riesgo en los grupos de 12 a 24 meses y $>24$ meses de tratamiento. Es notoria la mayor puntuación de riesgo en el grupo de 12-24 meses, siendo bajamedia en el grupo 0-12 meses y media en los grupos con mayor tiempo de tratamiento; aunque las calificaciones de riesgo son mayores las diferencias no son estadísticamente significativas $(p=0,608)$.

Esta evaluación analizó por separado las subescalas Ansiedad y Depresión y para ambas se encuentra una mayor calificación de riesgo en el grupo de 12-24 meses de tratamiento; estas diferencias no muestran significancia estadistica $(p=0,95$ y 0,49$)$. 
Tabla 2. Resultados puntuación escalas de riesgo del instrumento OTI

\begin{tabular}{|c|c|c|c|c|}
\hline \multirow[b]{2}{*}{ Variable } & \multicolumn{4}{|c|}{ Tiempo de tratamiento } \\
\hline & $\begin{array}{c}0-12 \text { meses } \\
n=21\end{array}$ & $\begin{array}{c}12-24 \text { meses } \\
n=15\end{array}$ & $\begin{array}{c}>24 \text { meses } \\
n=14\end{array}$ & $\begin{array}{c}\text { Total } \\
n=50\end{array}$ \\
\hline Puntuación escala, Drogas (incluye tabaco y alcohol) & 6,10 & 5,52 & 4,97 & 5,61 \\
\hline - Puntuación escala drogas, sin tabaco y alcohol & 0,57 & 2,22 & 0,88 & 1,15 \\
\hline - Puntuación escala drogas, sin tabaco & 0,81 & 2,24 & 0,90 & 1,26 \\
\hline - Puntuación escala drogas, sin alcohol & 5,87 & 5,49 & 4,95 & 5,50 \\
\hline Puntuación escala, conductas riesgo de contagio del VIH & $4,76(B-M)$ & $5,00(B-M)$ & $5,79(B-M)$ & $5,12(\mathrm{~B}-\mathrm{M})$ \\
\hline - Riesgo asociado al consumo de drogas & 0,86 & 1,20 & 1,36 & 1,10 \\
\hline - Riesgo asociado a la conducta sexual & 3,90 & 3,80 & 4,43 & 4,02 \\
\hline Puntuación escala, funcionamiento social & $13,43(B)$ & $11,13(B)$ & 11,64 (B) & $12,22(B)$ \\
\hline Puntuación escala, actividad delictiva & $0,10(B)$ & $0,00(B)$ & $0,29(B)$ & $0,12(B)$ \\
\hline Puntuación escala, estado de salud & $5,67(B)$ & $4,47(B)$ & $4,29(B)$ & $4,92(B)$ \\
\hline Puntuación escala ajuste psicológico GHQ-28 & $4,52(B-M)$ & $6,67(\mathrm{M})$ & $5,00(\mathrm{M})$ & $5,30(\mathrm{M})$ \\
\hline $\begin{array}{l}\text { - Ansiedad } \\
\text { - Depresión }\end{array}$ & $\begin{array}{l}1,57 \\
0,48\end{array}$ & $\begin{array}{l}1,80 \\
0,93\end{array}$ & $\begin{array}{l}1,57 \\
0,50\end{array}$ & $\begin{array}{l}1,64 \\
0,62\end{array}$ \\
\hline PUNTUACIÓN GLOBAL DEL OTI & 34,58 & 32,79 & 31,98 & \\
\hline
\end{tabular}

Categorias Clínicas de Gravedad para las puntuaciones de las Escalas del O.T.I.:

A: alta; M-A: media-alta; M: media; B-M: baja-media; B: baja

\section{Discusión}

La evaluación de resultados de tratamiento para las adicciones permiten generar sensibilidad pública y de proveedores de servicios de salud, ya que demuestran los beneficios en coste-efectividad que tienen los PMM para el tratamiento de la adicción a la heroína (Fernández, 2005). Evaluar los resultados de un PMM tiene relevancia para un país como Colombia, ya que históricamente las prevalencias de consumo de heroína no han sido allí altas (Castaño, 2002) y los proveedores de salud no tienen experiencia en la oferta de tratamientos efectivos; por ello, la descripción de resultados de experiencias existentes mejorará el conocimiento local sobre el tema y permitirá el ajuste de programas y protocolos nacionales; adicionalmente, si la medición de resultados de tratamiento se realiza con instrumentos validados a nivel internacional, se facilita la comparación de la situación local con experiencias internacionales.

La evaluación realizada en este primer estudio en Colombia, aunque con las limitaciones del tamaño de muestra, arrojó como resultado que el tratamiento de sustitución con Metadona ocurre en proporción hombre-mujer de 3:1 en una edad media de 23 años y edades de inicio tempranas; esta situación contrasta con otros estudios en Europa y en los Estados Unidos en donde las edades medias de pacientes en tratamiento son en su mayoría superiores a los 30 años (Fiellin, et al., 2001; Langendam, et al., 2001; Merril, Jackson, Schulman, Saxon, Awan, Kapitan, et al., 2005), lo cual lleva a concluir la posible mayor vulnerabilidad de jóvenes en esta región para el consumo y adicción a la heroína. Se destaca igualmente como la marihuana es la droga de inicio en el $70 \%$ de los individuos en este estudio y la cocaína en el 20\%; un porcentaje muy bajo inician el consumo de drogas con heroina, lo cual permite proponer que los esfuerzos de prevención deben fortalecerse de manera general en todas las sustancias psicoactivas y no de manera única o específica en la heroína.

Llama la atención que en el 38\%, 19 de los pacientes en este estudio se diagnosticó comorbilidad con otros trastornos mentales, correspondiendo a trastornos de personalidad cerca del 20\% (9) del total de la muestra. Este resultado es similar a los documentados por Sánchez et al. (1999), en donde se señala la alta prevalencia de trastornos de personalidad en adictos a opiáceos y al estudio de Gutiérrez, Saiz, González, Fernández y Bobes (1998) en el cual se afirma que el trastorno de personalidad antisocial es uno de los más frecuentes en esta población. Este hallazgo justifica la mayor cualificación de los servicios de tratamiento, dada la sofisticación terapéutica que se requiere para realizar intervenciones adecuadas para adictos a sustancias psicoactivas que presenten este tipo de comorbilidad (Fernández y Gutiérrez, 2005; Miller, 1996; 0’Connel, 1998).

Las dosis de metadona utilizadas son bajas comparadas con aquellas señaladas como efectivas por revisiones realizadas en el tema, las cuales sugieren valores entre 60 y $120 \mathrm{mg} /$ día (Krants y Mehler, 2004); sin embargo el rango máximo encontrado en este estudio para el tratamiento actual tiene niveles de $100 \mathrm{mg} /$ día aunque la mediana está en niveles de $40 \mathrm{mg}$. Esta situación puede obedecer al inicio de este programa en Colombia y al proceso normal de ganar experiencia y confianza en sus resultados; otra posible respuesta a esta situación puede ser la baja gravedad del proceso de adicción de los pacientes que maneja la institución en donde se realizó el estudio en Colombia.

La evaluación con el instrumento OTI en la escala consumo de drogas muestra una disminución general del riesgo del consumo de sustancias legales e ilegales a mayor periodo de tratamiento de sustitución con metadona, hallazgo similar al encontrado en el estudio por Fernández et al (2001); paralelamente se observa un mayor indicador de riesgo para el 
consumo de drogas en el periodo de 1 a 2 años de tratamiento, cuando se realiza análisis excluyendo el alcohol y tabaco; los valores de riesgo no difieren mucho cuando se realiza análisis excluyendo solamente el alcohol pero si cambian ostensiblemente cuando se excluye el tabaco, lo cual muestra una aparente concurrencia entre consumo de heroina y consumo de tabaco; para lo anterior se estima necesario un análisis que aborde especificamente el tema lo cual supera el alcance de este estudio.

Las escalas de riesgo del instrumento OTI para las conductas de riesgo de contagio de VIH mostraron un riesgo bajo en los tres grupos evaluados; aunque la variación en dicho riesgo no fue estadísticamente significativa, tuvo un ligero aumento en la calificación de riesgo a mayor tiempo de tratamiento. Esto apunta a la posible necesidad de incrementar acciones educativas y no disminuir su intensidad y frecuencia en el seguimiento de estos pacientes.

La escala de funcionamiento social muestra un riesgo bajo en la escala OTI para los grupos de pacientes estudiados; una disminución en la calificación numérica de este riesgo se observa en los periodos de tratamiento mayor a 12 meses, lo cual puede mostrar un ajuste progresivo positivo de los pacientes a su entorno social.

De igual manera, los indicadores de riesgo son cercanos a cero en la escala de riesgo actividad delictiva; este hallazgo puede apoyar la hipótesis que esta es una nueva población de consumidores, cuyo deterioro debido a su problema de adicción no llega a afectar aún de manera grave el comportamiento. La escala de salud muestra un riesgo bajo y descendente con el progreso del tiempo en tratamiento, pero la escala de ajuste psicológico muestra un riesgo que se incrementa en el periodo de 12 a 24 meses en donde tiene una categoria de gravedad media, lo cual lleva a pensar en la necesidad de fortalecer las intervenciones psicoterapéuticas durante este periodo.

De manera general puede concluirse que la población evaluada en este estudio es de bajo riesgo para las diferentes áreas del instrumento de medición utilizado; este bajo riesgo puede ser indicativo de que el problema de la adicción a heroína en nuestro país está en sus estadios iniciales y se convierte en prioritario fortalecer todas las acciones preventivas para que el problema no avance y para que aquellos con dependencia a la heroína y actualmente en tratamiento de sustitución, reciban la mejor atención disponible acorde a sus necesidades (March, Martin-Ruiz, Oviedo-Joekes, Rivadeneira y Rodríguez, 2006) y la mayor facilidad de acceso a los servicios vía inclusión de los PMM en los modelos de Atención Primaria en Salud disponibles o en desarrollo (Rodríguez, Millán, Venegas y March, 2010).

\section{Agradecimientos}

A la Institución de tratamiento CARISMA, a la Facultad de Medicina y la Dirección de Investigaciones de la Universidad CES quienes prestaron su apoyo para la realización de este estudio. Especial reconocimiento a los pacientes, a la
Médica Toxicóloga Ana Maria Vásquez por su participación en la recolección de la información y a las Gerentes en Sistemas de Información Viviana Castro y Gloria Maria Sierra por su dedicación en el procesamiento de datos.

\section{Conflicto de Intereses}

Los autores del artículo manifiestan no tener ningún conflicto de intereses.

\section{Referencias}

Adelekan, M., Green, A., Dasgupta, N., Tallack, F., Stimson, G.V., Wells, B. (1996). Reliability and validity of the Opiate Treatment Index among a sample of opioid users in the United Kingdom. Drug Alcohol Review, 15, 261-270.

American Psychiatric Association. DSM5 2010. Recuperado el 8 de Marzo de 2010, de: http://www.dsm5.org/Pages/Default.aspx

Castaño, A. (2002). Consumo de heroína en Colombia. Adicciones, 14, 87-90.

Castaño, G.A. y Calderón, G. A. (2010). Consumo de heroína en Colombia, prácticas relacionadas e incidencia en la salud pública. Revista Cubana de Salud Pública, 36, 311-322.

Correctional Service of Canada (2003). Specific Guidelines for Methadone Maintenance Treatment. Disponible en internet: www. csccc.gc.ca/text/pblct/methadone/index-eng.shtml

Darke, S., Hall, W., Wodak, A., Heather, N. y Ward, J. (1992). Development and validation of a multi-dimensional instrument for assessing outcome of treatment among opiate users: the Opiate Treatment Index. British Journal of Addiction, 87, 733-742.

Deering, D.E. y Sellman, J.D. (1996). An inter-rater reliability study of the Opiate Treatment Index (OTI). Drug and Alcohol Review, 15, 57-63.

Erickson, P.G. (1995). Harm Reduction: What it is and is not. Drug and Alcohol Review, 14, 283-285.

Fernández, J.J. (2005). Efectividad de los programas de mantenimiento con metadona. Adicciones, 17, 167-180.

Fernández J.J., González M.P., Saiz P.A., Gutiérrez E., Bobes, J. (2001). Consumo de drogas ilegales y alcohol antes y a lo largo de 6 años de mantenimiento con metadona y variables relacionadas. Adicciones, 13, 179-188.

Fernández, J.J. y Gutiérrez, E. (2005). Trastornos de personalidad y dependencia de heroina: una relación por determinar. Adicciones, 17,95-110.

Fiellin, D.A., O'Connor, P.G., Chawarski, M., Pakes, J.P., Pantalon, M.V. y Schottenfeld, R.S. (2001). Methadone Maintenance in Primary Care. A Randomized Controlled Trial. Journal of American Medical Association, 286, 1724-1731.

Goldberg, D. y Williams, P. (1988). A user's guide to the General Health Questionnaire. NFER NELSON Publishing Company Ltd. Windsor.

González-Saiz, F.M., Salvador-Carulla, L., Martínez-Delgado, J.M., López-Cárdenas, A., Ruz-Franzi, I. y Guerra-Díaz, D. (1997). Indicador del Tratamiento de la Adicción a Opiáceos. Ed. Servicio de Publicaciones de la Universidad de Cádiz.

Gutiérrez, E., Saiz, P., González, M.P., Fernández, J.J. y Bobes, J. (1998). Trastornos de personalidad en adictos a opiáceos en tratamiento con agonistas vs antagonistas. Adicciones, 10, 121-129. 
Henry-Edwards, S., Gowing, L., White, J., Ali, R., Bell, J., Brough, R. et al. (2003). Clinical Guidelines and Procedures for the Use of Methadone in the Maintenance Treatment of Opioid Dependence. Publication Production Unit, Australian Government Department of Health and Ageing. Disponible en internet: http://www.dassa. sa.gov.au/webdata/resources/files/Methadone_cguide_abbrev.pdf

Krantz, M.J., Mehler, P.S. (2004). Treating Opioid Dependence, Growing Implications for Primary Care. Archives of Internal Medicine, 164, 277-288.

Langendam, M.W., van Brussel G.H., Coutinho, R.A. y van Ameijden, E.J. (2001). The impact of harm-reduction-based Methadone treatment on Mortality among Heroin users. American Journal of Public Health, 91, 774-780.

March, J.C., Martin-Ruiz, E., Oviedo-Joekes, E., Rivadeneira, A. y Rodríguez, C. (2006). Percepción de usuarios de los Programas de Tratamiento con Metadona sobre la accesibilidad y atención recibida de los equipos terapéuticos. Adicciones, 18, 359-370.

Merrill, J.0., Jackson, T.R., Schulman, B.A., Saxon, A.J., Awan, A., Kapitan, S., et al., (2005). Methadone Medical Maintenance in Primary Care, an Implementation Evaluation. Journal of General Internal Medicine, 20,344-349.

Miller, N. (1996). Issues in the diagnosis and treatment of comorbid addictive and other psychiatric disorders. Addiction psychiatry: Current diagnosis and treatment. New York.

NIH Consensus Conference (1998). Effective Medical Treatment of Opiate Addiction. Journal of American Medical Association, 280, 1936-1943.

O'Connell, D. (1998). Dual disorders: essentials for assessment and treatment. The Haword Press. New York.
Rodríguez, C., Millán, A., Venegas, J. y March, J.C. (2010). Oportunidades de mejora del servicio de metadona en atención primaria, desde el punto de vista profesional. Adicciones, 22, 207216.

Sánchez, E., Tomas, V., Climent, A. (1999). Trastornos de personalidad en adictos a opiáceos. Adicciones, 11, 221-227.

Tarter, R., Kirisci, L. y Mezzich, A. (1996). The Drug Use Screening Inventory: School Adjustment Correlates of Substance Abuse. Measurement and Evaluation in Counseling and Development, 29, 25-34

Trujols, J., Iraurgi, I., Sola, I., Ballesteros, J., Siñol, N., Battle, F. y Pérez, J. (2010). Infección por VIH y usuarios de drogas por vía parenteral: urgencia de la revitalización de los programas de reducción de daño. Adicciones, 22, 135-140.

UNODC (2006). Proyecto Treatnet fase I. Recuperado el 24 de Febrero de 2011, de: http://www.unodc.org/treatment/en/drug-treatmentnetwork---home-archive.html

United Nations Office on Drugs and Crime - UNODC (2004). World Drug Report 2004. Volume 1: Analysis. Recuperado el 9 de Diciembre de 2010, de http://www.unodc.org/pdf/WDR_2004/ volume_1.pdf

United Nations Office on drugs and Crime - UNODC (2010). World Drug Report 2010. Recuperado el 27 de Abril de 2011, de http:// www.unodc.org/documents/wdr/WDR_2010/World_Drug_ Report_2010_lo-res.pdf

Zapata, M. A. (2004). Heroína, Amenaza de Epidemia. Periódico El Mundo, Medellín, Colombia, 11 de Octubre de 2004. 
